\title{
Localisation de la vorticité et applications au comportement asymptotique des solutions de Navier-Stokes
}

\author{
Lorenzo Brandolese
}

\section{Résumé}

Nous présentons quelques résultats de localisation, en variables d'espace, temps et fréquence, pour la vorticité associée aux écoulements dans $\mathbb{R}^{3}$ d'un fluide visqueux. Nous étudions, ensuite, la localisation des écoulements caractérisés par des fortes oscillations de la vorticité.

\begin{abstract}
We study the localization, with respect to the space, time and frequency variables, of the vorticity of a viscous flow filling the whole space. The special behavior of flows with highly oscillating vorticity is also discussed.
\end{abstract}

\section{Introduction}

Nous allons étudier le mouvement d'un fluide visqueux, incompressible, remplissant l'espace $\mathbb{R}^{3}$ tout entier et dont la vorticité initiale est localisée. L'écoulement est gouverné par les équations de Navier-Stokes qui, en termes de la vorticité $\omega=$ $\left(\omega_{1}, \omega_{2}, \omega_{3}\right)$, peuvent s'écrire

$$
\begin{aligned}
& \partial_{t} \omega-\Delta \omega+\sum_{h=1}^{3} \partial_{h}\left(u_{h} \omega-\omega_{h} u\right)=0, \\
& \omega(0)=\mu, \quad \operatorname{div}(\omega)=0 .
\end{aligned}
$$

Ici

$$
u(x, t)=-\frac{1}{4 \pi} \int \frac{x-y}{|x-y|^{3}} \wedge \omega(y, t) d y
$$

est le champ de vitesse et

$$
\omega=\nabla \wedge u=\left(\partial_{2} u_{3}-\partial_{3} u_{2},-\partial_{1} u_{3}+\partial_{3} u_{1}, \partial_{1} u_{2}-\partial l_{2} u_{1}\right) .
$$

Nous allons présenter un résultat de propagation de la localisation spatiale de $\omega$, ainsi qu'un résultat de localisation fréquentielle. Le premier sera obtenu de façon 
simple et directe à l'aide de quelques estimation de décroissance pour $|x| \rightarrow \infty$. Les résultats de localisation fréquentielle montrent que, sous des conditions convenables, le profil de $\widehat{\omega}(\xi, t)$ "ressemble à celui d'une ondelette" : $|\widehat{\omega}(\xi, t)|$ est porté pour l'essentiel par une couronne compacte, centrée en $\xi=0$. Un problème relié à ce dernier, que nous allons étudier en détail notamment dans le cas d'une classe particulière d'écoulements, est celui de la conservation des moments de $\omega$ au cours de l'évolution.

Le deuxième but de cet article est l'étude du comportement asymptotique, pour $t \rightarrow \infty$. Notre résultat principal implique l'existence de solutions telles que

$$
|\omega(x, t)| \leq C_{\alpha}(1+|x|)^{-\alpha}(1+t)^{-(S-\alpha) / 2}, \quad \text { pour tout } 0 \leq \alpha \leq \gamma
$$

où $\gamma$ et $S$ sont deux paramètres qui dépendent de la localisation spatiale de $\mu$, $S$ étant relié aussi au caractère oscillant et aux symétries de l'écoulement. Enfin, nous présentons une application de ces résultats à un problème classique sur le comportement asymptotique de l'énergie.

\section{Localisation en espace et en fréquence des solutions locales}

\subsection{Localisation spatiale}

Nous allons utiliser la forme intégrale de (NS) :

$$
\begin{array}{r}
\omega(t)=e^{t \Delta} \mu-A(u, \omega)(t), \quad \operatorname{div}(\mu)=0 \\
A(u, \omega)(t)=\int_{0}^{t} e^{(t-s) \Delta} \partial_{h}\left(u_{h} \omega-\omega_{h} u\right)(s) d s \\
u(t)=K * \omega(t)=-\frac{1}{4 \pi} \int \frac{x-y}{|x-y|^{3}} \wedge \omega(y, t) d y,
\end{array}
$$

où la dernière équation (loi de Biot-Savart) permet de retrouver le champ de vitesse à partir de sa vorticité, sous des conditions assez générales. Ici et par la suite la somme sur l'indice $h$ est omise.

Introduisons d'abord quelques notations: pour $\gamma \geq 0$ et $\gamma \neq 1$ on note par $Y_{\gamma}$, l'espace des fonctions $f$ (scalaires ou vectorielles), définies sur $\mathbb{R}^{3}$ et telles que $(1+|x|)^{\gamma} f(x) \in L^{\infty}\left(\mathbb{R}^{3}\right)$. On complète cette définition dans le cas $\gamma=1$, en posant $f \in Y_{1}$ si et seulement si $f \in L^{\infty}\left(\mathbb{R}^{3}\right)$ et s'il existe une suite $\epsilon_{j} \in \ell^{1}(\mathbb{N})$ telle que $|f(x)| \leq 2^{-j} \epsilon_{j}\left(2^{j} \leq|x|<2^{j+1}\right)$. Cet espace est normé par

$$
\|f\|_{Y_{1}}=\sup _{|x| \leq 1}|f(x)|+\sum_{j=0}^{\infty} 2^{j} \sup _{2^{j} \leq|x|<2^{j+1}}|f(x)| .
$$

Ensuite, si $\omega \in C\left([0, T], Y_{\gamma}\right)$, on exprime la continuité en dehors de 0 au sens naturel de la norme de l'espace et, à l'origine, par $\omega(t) \rightarrow \omega(0)$ au sens des distributions. On indique par $\|\cdot\|_{\gamma}$ la norme naturelle de $C\left([0, T], Y_{\gamma}\right)$.

Nous commençons par un résultat sur la propagation de la localisation spatiale de la vorticité (théorème 1, i)). Il s'agit là d'un phénomène expérimentalement bien connu et qui a été déjà traité dans des contextes un peu différentes, par exemple, 
dans [LT], [Da] et [GW]. Comme nous allons le voir, l'utilisation des espaces $L^{\infty}$ à poids nous permet d'en donner une description simple et directe. Quant à la troisième partie de ce théorème, elle est une première approche aux questions de localisation fréquentielle.

Théorème $1 \quad$ i) Soit $\mu \in Y_{1}$ et $\operatorname{div}(\mu)=0$. Il existe un temps $T>0$ et une unique solution $\omega \in C\left([0, T], Y_{1}\right)$ de $(\mathrm{EI})$. En outre, si $\gamma>1$ et si $\mu$ appartient aussi à $Y_{\gamma}$, alors $\omega \in C\left([0, T], Y_{\gamma}\right)$.

ii) Si $\gamma \neq 2,3,4$, on a de plus $u \in C\left([0, T], Y_{\tilde{\gamma}}\right)$, ò̀ $\tilde{\gamma}=\min \{3, \gamma-1\}$. En outre, si $\gamma>4, \gamma \neq 5$ et $\int x_{j} \mu(x) d x=0(j=1,2,3)$, alors $\int x_{j} \omega(x, t) d x=0$ pour tout $t \geq 0$. Dans ce cas, on peut prendre $\tilde{\gamma}=\min \{4, \gamma-1\}$.

iii) Si la vorticité initiale est à décroissance rapide : $|\mu(x)| \leq C_{m}(1+|x|)^{-m}$ $(m=1,2, \ldots)$, alors $\omega(t) \in \mathcal{S}\left(\mathbb{R}^{3}\right)$ (la classe de Schwartz) pour tout $\left.\left.t \in\right] 0, T\right]$. En outre, $\omega \in C\left([0, T], \mathcal{S}\left(\mathbb{R}^{3}\right)\right)$, si $\mu \in \mathcal{S}\left(\mathbb{R}^{3}\right)$.

Remarques. La conclusion ii) (dans le cas $\gamma>5$ et sous l'hypothèse de nullité des moments d'ordre 1 de $\mu$ ) permet de retrouver le résultat bien connu de propagation de la condition $u(x, 0)=O\left(|x|^{-4}\right)(x \rightarrow \infty)$. Ce taux de décroissance est d'ailleur optimal, dans le cas générique. La décroissance spatiale de $u$ étant reliée au nombre des moments nuls de $\omega$, on retrouve immédiatement, grâce aux résultats de diffusion instantanée pour le champ de vitesse (cf. [Bra01], [DS]), que les moments d'ordre deux de $\omega$ ne sont pas des invariants. La conservation des moments d'ordre 1 pour $\omega$ est aussi bien connue (cf. [LT]).

La deuxième partie du théorème est une conséquence immédiate de la première et de la loi de Biot-Savart. Si $\omega \in Y_{\gamma}(\gamma \geq 1)$, et $K(x)=-(4 \pi)^{-1} x /|x|^{3}$, alors $u=K * \omega$ a bien un sens et $u \in L^{\infty}\left(\mathbb{R}^{3}\right)$. La condition $u \in Y_{\tilde{\gamma}}$ s'obtient sans peine, pour $1 \leq \gamma<2$ et $2<\gamma<3$ à l'aide de découpages élémentaires dans l'intégrale de convolution. Dans le cas $\gamma>3$ on utilise $\int \omega=0$ (c'est une conséquence de la condition de divergence nulle) ; il suffit alors d'intégrer par parties et d'utiliser $|\nabla K(x)| \leq C|x|^{-3}$.

Si $\gamma=2$, on a $(1+|x|)|u(x, t)| \in L^{\infty}\left(\mathbb{R}^{3}\right)$. Dans les cas $\gamma=3,4$ ou 5 , on ne peut obtenir qu'une estimation de la forme $(1+|x|)^{\gamma-1}|u(x, t)| \leq C \log (2+|x|)$.

La solution de (EI) sera construite à l'aide du schéma usuel

$$
\begin{aligned}
& \omega^{j+1}(t)=e^{t \Delta} \mu-A\left(u^{j}, \omega^{j}\right)(t) \\
& \omega^{0}(t)=e^{t \Delta} \mu \\
& u^{j}(t)=K * \omega^{j}(t), \quad j=0,1,2, \ldots
\end{aligned}
$$

Le lemme suivant assure la convergence de $\left(u^{j}, \omega^{j}\right)$ dans $C\left(\left[0, T_{\gamma}\right], Y_{\tilde{\gamma}}\right) \times C\left(\left[0, T_{\gamma}\right], Y_{\gamma}\right)$, au moins pour $T_{\gamma}>0$ assez petit.

Lemme 1 Soit $T>0, \gamma \geq 1$ et $\omega \in C\left([0, T], Y_{\gamma}\right)$. On pose $u=K * \omega$ et on définit $A(u, \omega)$ comme dans (EI). On a alors

$$
A(u, \omega) \in C\left([0, T], Y_{\gamma}\right),
$$

avec $\|A(u, v)\|_{\gamma} \leq C_{\gamma} C_{T}\|\omega\|_{\gamma}^{2}$ et $C_{T}=O\left(T^{1 / 2}\right)$ pour $T \rightarrow 0$. 
Dém. Dans ce qui suit, on utilise simplement $u \in C\left([0, T], Y_{0}\right)$. On peut supposer, sans restriction, $\|\omega\|_{\gamma}=1$. Observons que pour tout $\alpha \geq 0$, on peut trouver des constantes positives $c$ et $C_{\alpha}$ telles que le noyau $f(x, t)$ de l'opérateur $\nabla e^{t \Delta}$ vérifie $\|f(\cdot, t)\|_{1}=c t^{-1 / 2}$ et $|f(x, t)| \leq C_{\alpha}|x|^{-\alpha} t^{(\alpha-4) / 2}$. Par conséquent, $\|A(u, \omega)(t)\|_{\infty} \leq$ $C T^{1 / 2}$. On peut se limiter donc à $|x| \geq 1$.

Commençons par traiter le cas $1 \leq \gamma \leq 4$. En choisissant $\alpha=4$, on voit que si $I(x, s, t) \equiv \int f(x-y, t-s) \omega(y, s) u(y, s) d y$, alors

$$
|I(x, s, t)| \leq C|x|^{-4} \int_{|y| \leq|x| / 2}|\omega(y, s)| d y+C(t-s)^{-1 / 2} \sup _{|y| \geq|x| / 2}|\omega(y, s)| .
$$

Si $\gamma=1$ et $2^{j} \leq|x| \leq 2^{j+1}(j \in \mathbb{N})$, on a $|A(u, \omega)(x, t)| \leq C T|x|^{-2}+C T^{1 / 2} 2^{-j} \epsilon_{j}$, avec $\epsilon_{j} \in \ell^{1}$. Si $1<\gamma \leq 4(\gamma \neq 3)$ on a $|A(u, \omega)(x, t)| \leq C T|x|^{-\delta}+C T^{1 / 2}|x|^{-\gamma}$, où $\delta=\min \{4, \gamma+1\}$.

Supposons maintenant $\gamma>4$. On majore $I(x, t, s)$ en prenant $\alpha=\gamma$ et l'on obtient $|A(u, \omega)(x, t)| \leq C T^{(\gamma-2) / 2}|x|^{-\gamma}+C T^{1 / 2}|x|^{-\gamma}$.

Dans tous les cas, on a bien $A(u, \omega)(t) \in Y_{\gamma}$ et la démonstration de la continuité en $t$ est triviale.

Les estimations du terme linéaire $e^{t \Delta} \mu$ sont faciles et l'on trouve sans aucune difficulté que, si $T=T_{\gamma}$ est assez petit, on a

$$
\sup _{t \in[0, T]}(1+|x|)^{\gamma}\left|e^{t \Delta} \mu(x)\right|<1 /\left(4 C_{\gamma} C_{T}\right),
$$

où $C_{\gamma}$ et $C_{T}$ sont les constantes du lemme 1.

Il est bien connu que (1.3) assure la convergence de l'algorithme (1.2). On voit alors que $\left(u^{j}, \omega^{j}\right)$ converge vers une solution $(u, \omega)$ de $(\mathrm{EI})$, unique dans $C\left(\left[0, T_{\gamma}\right], Y_{0}\right) \times$ $C\left(\left[0, T_{\gamma}\right], Y_{\gamma}\right)$. Ici, $T_{\gamma}$ est minoré par $C\|\mu\|_{Y_{\gamma}}^{-1 / 2}$. En particulier, $\omega(t)$ conserve, au moins au début de son évolution, la localisation de la donnée initiale.

Il reste à démontrer que le taux de décroissance de $\omega(x, t)$ ne se dégrade pas au cours du temps: si $\mu \in Y_{\gamma}(\gamma>1)$, alors $T_{\gamma}=T_{1}$ pour tout $\gamma>1$.

Pour ce faire, il suffit de montrer que si $T<T_{1}$ est fixé de façon que $\omega \in$ $\cap_{\tau<T} C\left([0, \tau], Y_{\gamma}\right)$, alors $\|\omega(t)\|_{Y_{\gamma}}$ est uniformément borné sur $t \in[0, T[$.

En effet, pour $0 \leq t<T,\|\omega(t)\|_{Y_{\gamma}} \leq C_{1}(T)+C_{2}(T) \int_{0}^{t}(t-s)^{-1 / 2}\|\omega(s)\|_{Y_{\gamma}} d s$. Si on majore $\|\omega(s)\|_{Y_{\gamma}}$ en réiterant une fois cette inégalité, on en déduit $\|\omega(t)\|_{Y_{\gamma}} \leq$ $C_{3}(T)+C_{4}(T) \int_{0}^{t}\|u(\sigma)\|_{Y_{\gamma}} d \sigma$. D'après l'inégalité de Gronwall, $\|\omega(t)\|_{Y_{\gamma}} \leq C_{3}(T) e^{C_{4}(T) t}$.

La première et la deuxième partie du théorème 1 sont ainsi prouvées. Nous allons obtenir la démonstration de la troisième partie du théorème en guise de corollaire de quelques résultats de localisation fréquentielle.

\subsection{Localisation fréquentielle}

Nous allons obtenir ici des estimations de décroissance pour la transformée de Fourier de la vorticité. Le résultat de la proposition 1 ci dessous implique que la solution construite dans la section précédente vérifie

$$
|\widehat{\omega}(\xi, t)| \leq C_{m}(t)(1+|\xi|)^{-m} \quad(m=1,2, \ldots)
$$


pour tout $t(0<t \leq T)$. Ceci, et la remarque $\widehat{\omega}(0, t)=0$ et $\nabla \widehat{\omega}(0, t)=0$ (cette dernière condition étant assurée si $\left.\int x_{j} \mu(x) d x=0\right)$ justifient le titre de cette section. Par ailleurs, dans la section 4 nous verrons que pour certains écoulements particuliers, $\widehat{\omega}(\xi, t)$ s'annulle en $\xi=0$ d'un ordre assez élevé. Évidemment les constantes $C_{m}(t)$ vont exploser, en général, lorsque $t \rightarrow 0$ (on aurait explosion également pour une viscosité du fluide évanescente, (1.4) étant intimément reliée à la nature parabolique de l'équation et à son effet régularisant), à moins de supposer que $\widehat{\mu}$ soit elle même à décroissance rapide pour $\xi \rightarrow \infty$.

Nous commençons par réécrire la loi de Biot-Savart sous la forme $\widehat{u}(\xi, t)=$ $\widehat{K}(\xi) \widehat{\omega}(\xi, t)=c|\xi|^{-1} \widehat{\omega}(\xi, t)$. Sous les conditions du théorème 1, iii) (mais $\omega(t) \in Y_{\gamma} \subset$ $L^{1}\left(\mathbb{R}^{3}\right),(\gamma>4)$, serait suffisant $), \widehat{\omega}$ et $\widehat{u}$ appartiennent évidemment à $C\left([0, T], L^{2}\left(\mathbb{R}^{3}\right) \cap\right.$ $\left.L^{\infty}\left(\mathbb{R}^{3}\right)\right)$.

Lorsque $\mu$ appartient de plus à $\mathcal{F} L^{1}\left(\mathbb{R}^{3}\right)$ (l'algèbre de Wiener), on a un peu mieux :

Lemme 2 Soient $\mu \in Y_{\gamma}(\gamma>4), \operatorname{div}(\mu)=0$ et $\omega \in C\left([0, T], Y_{\gamma}\right)$ la solution obtenue dans la le theorème 1. Si $\mu \in \mathcal{F} L^{1}\left(\mathbb{R}^{3}\right)$, on peut trouver $T^{\prime}\left(0<T^{\prime} \leq T\right)$, tel que $\omega \in C\left(\left[0, T^{\prime}\right], L^{1}\left(\mathbb{R}^{3}\right) \cap \mathcal{F} L^{1}\left(\mathbb{R}^{3}\right)\right)$ et $u \in C\left(\left[0, T^{\prime}\right], L^{1}\left(\mathbb{R}^{3}\right) \cap \mathcal{F} L^{1}\left(\mathbb{R}^{3}\right)\right)$.

La démonstration de ce lemme est très simple et nous la laissons au lecteur. Mais nous voulons prendre en compte aussi de vorticités initiales discontinues : si $\mu \notin \mathcal{F} L^{1}\left(\mathbb{R}^{3}\right)$, nous exploitons l'effet régularisant de (EI) et nous attendons un temps $\epsilon>0$, par ailleurs arbitrairement >petit, de façon à avoir $\omega \in C\left(\left[\epsilon, T^{\prime \prime}\right], L^{1}\left(\mathbb{R}^{3}\right) \cap\right.$ $\left.\mathcal{F} L^{1}\left(\mathbb{R}^{3}\right)\right)$ et $u \in C\left(\left[\epsilon, T^{\prime \prime}\right], L^{1}\left(\mathbb{R}^{3}\right) \cap \mathcal{F} L^{1}\left(\mathbb{R}^{3}\right)\right)\left(T^{\prime \prime}>0\right)$. Pour le voir, on fixe $0<\epsilon<$ $T$ et on commence par l'observer que $|\widehat{\omega}(\xi, t)| \leq e^{-t|\xi|^{2}}|\widehat{\omega}(\xi, \epsilon)|+\int_{\epsilon}^{t}|\xi| e^{-(t-s)|\xi|^{2}}(|\widehat{u}| *$ $|\widehat{\omega}|)(\xi, s) d s$. Ensuite il suffit d'appliquer le lemme suivant :

Lemme 3 Soit $T>0$ et $0 \leq t \leq T$. On pose $F(t)=\int_{0}^{t}|\xi| e^{-(t-s)|\xi|^{2}}\left(\frac{1}{|\cdot|} f\right) * f(\xi) d s$. Alors

i) Si $f \in L^{2} \cap L^{\infty}$, on a $F \in C\left([0, T], L^{p_{1}}\right)$, avec $3 / 2<p_{1} \leq \infty$

ii) Si $f \in L^{5 / 3} \cap L^{\infty}$, on a $F \in C\left([0, T], L^{p_{2}}\right)$, avec $15 / 13<p_{2} \leq \infty$

iii) Si $f \in L^{4 / 3} \cap L^{\infty}$, on a $F \in C\left([0, T], L^{1} \cap L^{\infty}\right)$.

Démontrons i). Pour tout $\delta>0$, on a

$$
|\xi| e^{-(t-\cdot)|\xi|^{2}} \in L^{1}\left([0, t], L^{3+\delta} \cap L^{\infty}\right) .
$$

En outre, comme $f$ et $(1 /|\cdot|) f$ appartiennent à $L^{2}$, on a $(1 /|\cdot|) f * f \in L^{\infty}$ et $F \in$ $C\left([0, T], L^{\infty}\right)$. Si l'on observe que $1 /|\cdot| \in L^{1}+L^{3+\delta}$, les inégalités de Hölder et de Young entraînent, cette fois,

$$
(1 /|\cdot|) f \in L^{1}+L^{6 / 5+\delta}, \quad \text { et } \quad(1 /|\cdot|) f * f \in L^{2}+L^{3+\delta},
$$

et donc $F \in C\left([0, T], L^{3 / 2+\delta}\right)$, pour tout $\delta>0$.

La démonstration de ii) et iii) suit les mêmes lignes et est laissée au lecteur. 
En appliquant, de façon iterative, i), ii) et iii) de ce lemme (avec $f=|\widehat{\omega}(s)|$ et $(1 /|\cdot|) f=|\widehat{u}(s)|)$, on trouve que $\widehat{\omega}(t)$ et $\widehat{u}(t)$ appartiennent à $L^{1}\left(\mathbb{R}^{3}\right) \cap L^{\infty}\left(\mathbb{R}^{3}\right)$ uniformément dans $[\epsilon, T]$, pour tout $\epsilon>0$.

Nous pouvons maintenant établir (1.4). L'estimation de la proposition suivante est plus précise et permet de mesurer, de quelques sortes, le "déplacement vers les basses fŕequences" au cours du temps.

Proposition 1 Soit $(u, \omega)$ une solution de (EI) avec $\omega(0)=\mu$, définie sur un intervalle $[0, T]$, telle que l'on ait

$$
\begin{aligned}
\lambda_{1} & \equiv \sup _{t \in[0, T]}\|\widehat{u}(t)\|_{1}+\|\widehat{\omega}(t)\|_{1}<\infty \\
\lambda_{\infty} & \equiv \sup _{t \in[0, T]}\|\widehat{u}(t)\|_{\infty}+\|\widehat{\omega}(t)\|_{\infty}<\infty
\end{aligned}
$$

On note par $\mathcal{M}(\xi)$ la plus petite majorante radiale et décroissante de $\widehat{\mu}(\xi)$ :

$$
\mathcal{M}(\xi)=\sup _{|\eta| \geq|\xi|}|\widehat{\mu}(\eta)|
$$

Alors, pour tout $m=1,2, \ldots$ et $0 \leq t \leq T$, on a

$$
|\widehat{\omega}(\xi, t)| \leq C_{m}\left[\Phi_{m}(|\xi|, t)+\frac{\lambda_{1}^{m} \lambda_{\infty} t^{m / 4}}{|\xi|^{m / 2}}\right] \quad \text { si }|\xi| \geq 2^{m-1},
$$

où $C_{m}$ ne dépend que de $m$. Ici on a posé

$$
\begin{aligned}
\Phi_{m}(|\xi|, t)=\exp \left(-t|\xi|^{2}\right)|\widehat{\mu}(\xi)| & +\lambda_{1} \mathcal{M}\left(\frac{\xi}{2}\right) \exp \left(-\frac{t|\xi|^{2}}{4}\right) t^{1 / 2} \\
& +\cdots+\lambda_{1}^{m-1} \mathcal{M}\left(\frac{\xi}{2^{m-1}}\right) \exp \left(-\frac{t|\xi|^{2}}{4^{m-1}}\right) t^{(m-1) / 2}
\end{aligned}
$$

Remarques. Le premier terme à droite dans (1.7) a une décroissance exponentielle en $\xi$, si $t>0$, et le second une décroissance algébrique, mais arbitrairement elévée.

Supposons $\mu \in Y_{\gamma}$, avec $\gamma>4$. D'après les remarques précédentes, la solution $(u, \omega)$ vérifie les hypothèses de la proposition sur tout intervalle $[\epsilon, T](\epsilon>0)$. En particulier, $\omega$ vérifie (1.7), avec $C_{m}=C_{m}(t)$, et $\widehat{\omega}(t)$ est à décroissance rapide, pour $\xi \rightarrow \infty$, si $0<t \leq T$. Comme nous l'avons observé si, de plus, $\widehat{\mu} \in L^{1}\left(\mathbb{R}^{3}\right)$, alors $C_{m}(t) \leq C<\infty$ pour tout $t \in[0, T]$

2. Il est à noter que la propriété de localisation fréquentielle (1.6) permet déjà de donner un sens à l'équation intégrale

$$
\begin{aligned}
& \widehat{\omega}(\xi, t)=e^{-t|\xi|^{2}} \widehat{\mu}(\xi)+i \int_{0}^{t} \xi_{h} e^{-(t-s)|\xi|^{2}}\left(\widehat{u_{h} \omega}-\widehat{\omega_{h} u}\right)(\xi, s) d s \\
& \widehat{u}(\xi, t)=\widehat{K}(\xi) \wedge \widehat{\omega}(\xi, t), \quad \xi \cdot \widehat{\mu}(\xi)=0 .
\end{aligned}
$$

C'est ainsi que nous formalisons, ici la notion de solution. 
Dém. Commençons par le cas $m=1$, en supposant, donc, $|\xi| \geq 1$. D'après (1.9), on a

$$
|\widehat{\omega}(\xi, t)| \leq e^{-t|\xi|^{2}}|\widehat{\mu}(\xi)|+\int_{0}^{t}|\xi| e^{-(t-s)|\xi|^{2}}(|\widehat{u}| *|\widehat{\omega}|)(\xi, s) d s .
$$

Comme $|\widehat{u}| *|\widehat{\omega}|(\xi, t) \leq \lambda_{\infty} \lambda_{1}$, on obtient

$$
\begin{aligned}
|\widehat{\omega}(\xi, t)| \leq e^{-t|\xi|^{2}}|\widehat{\mu}(\xi)|+\lambda_{\infty} \lambda_{1} \int_{0}^{t}|\xi| e^{-(t-s)|\xi|^{2}} d s & \\
& \leq e^{-t|\xi|^{2}}|\widehat{\mu}(\xi)|+c \lambda_{\infty} \lambda_{1}|\xi|^{-1 / 2} t^{1 / 4}
\end{aligned}
$$

Afin de mieux illustrer la démonstration, avant de procéder par récurrence, montrons explicitement le résultat aussi pour $m=2$. D'après (1.11) et la loi de Biot-Savart, on voit que $|\widehat{\omega}(\xi, t)|$ et $|\widehat{u}(\xi, t)|$ sont majorées par $\mathcal{M}(\xi) e^{-t|\xi|^{2}}+$ $c \lambda_{\infty} \lambda_{1}|\xi|^{-1 / 2} t^{1 / 4}$, si $|\xi| \geq 1$ et $0 \leq t \leq T$. (pour $\widehat{u}$ on aurait, en fait, un facteur $|\xi|^{-1}$ de mieux).

Procédons maintenant avec l'estimation de $|\widehat{u}| *|\widehat{\omega}|(\xi, t)$. Pour $|\xi| \geq 2$,

$$
\begin{aligned}
|\widehat{u}| *|\widehat{\omega}|(\xi, t) \leq\left(\int_{|\eta| \leq|\xi| / 2}+\right. & \left.\int_{|\eta| \geq|\xi| / 2}\right)|\widehat{u}(\xi-\eta, t)||\widehat{\omega}(\eta, t)| d \eta \\
& \leq 2 \lambda_{1} \mathcal{M}(\xi / 2) e^{-t|\xi|^{2} / 4}+2 \sqrt{2} c \lambda_{\infty} \lambda_{1}^{2}|\xi|^{-1 / 2} t^{1 / 4} .
\end{aligned}
$$

On revient maintenant à (1.10) et on déduit

$$
\begin{aligned}
|\widehat{\omega}(\xi, t)| \leq e^{-t|\xi|^{2}}|\widehat{\mu}(\xi)|+2 \lambda_{1} \mathcal{M}(\xi / 2) \int_{0}^{t}|\xi| e^{(-t+3 s / 4)|\xi|^{2}} d s & \\
& +2 \sqrt{2} c \lambda_{\infty} \lambda_{1}^{2} \int_{0}^{t}|\xi|^{1 / 2} e^{-(t-s)|\xi|^{2}} s^{1 / 4} d s .
\end{aligned}
$$

On a, par exemple, $\int_{0}^{t}|\xi| e^{(-t+3 s / 4)|\xi|^{2}} d s \leq \frac{2}{\sqrt{3}} e^{-t|\xi|^{2} / 4} t^{1 / 2}$, comme l'on voit par interpolation sur les deux majorations immédiates $\frac{4}{3}|\xi|^{-1} e^{-t|\xi|^{2} / 4}$ et $|\xi| e^{-t|\xi|^{2} / 4} t$ de cette intégrale.

Donc,

$$
|\widehat{\omega}(\xi, t)| \leq C\left[e^{-t|\xi|^{2}}|\widehat{\mu}(\xi)|+\lambda_{1} \mathcal{M}(\xi / 2) e^{-t|\xi|^{2} / 4} t^{1 / 2}+\lambda_{1}^{2} \lambda_{\infty}|\xi|^{-1} t^{1 / 2}\right], \quad|\xi| \geq 2
$$

et la conclusion est vraie, si $m=2$.

Supposons (1.7) vraie pour $m$ et prouvons-la pour $m+1$. La première observation triviale est que $|\widehat{\omega}(\xi, t)|$ et $|\widehat{u}(\xi, t)|$ sont majorées, uniformément sur $[0, T]$, par

$$
C_{m}\left[\tilde{\Phi}_{m}(|\xi|, t)+\frac{\lambda_{1}^{m} \lambda_{\infty} t^{m / 4}}{|\xi|^{m / 2}}\right], \quad \text { si }|\xi| \geq 2^{m-1} .
$$

Ici $\tilde{\Phi}$ coïncide avec $\Phi$, à ceci près que dans (1.8) on remplace $|\widehat{\mu}(\xi)| \operatorname{par} \mathcal{M}(\xi)$. Comme $\rho \mapsto \tilde{\Phi}_{m}(\rho, t)$ est décroissante en $\rho>0$, pour tout $t \geq 0$, nous pouvons appliquer la même méthode de l'étape précedente, pour estimer $|\widehat{u}| *|\widehat{\omega}|(\xi, t)$. On obtient facilement

$$
|\widehat{u}| *|\widehat{\omega}|(\xi, t) \leq C_{m}\left[\lambda_{1} \tilde{\Phi}_{m}(|\xi| / 2, t)+\lambda_{1}^{m+1} \lambda_{\infty}(|\xi| / 2)^{-m / 2} t^{m / 4}\right] .
$$


L’inégalité (1.10) entraîne alors

$$
\begin{aligned}
|\widehat{\omega}(\xi, t)| \leq e^{-t|\xi|^{2}}|\widehat{\mu}(\xi)|+C_{m}^{\prime} \lambda_{1} \int_{0}^{t}|\xi| & e^{-(t-s)|\xi|^{2}} \tilde{\Phi}_{m}(|\xi| / 2, s) d s \\
& \quad+C_{m}^{\prime} \lambda_{1}^{m+1} \lambda_{\infty} \int_{0}^{t}|\xi| e^{-(t-s)|\xi|^{2}}|\xi|^{-m / 2} s^{m / 4} d s
\end{aligned}
$$

La seconde intégrale est majorée de façon triviale par $C_{m+1} \lambda_{\infty} \lambda_{1}^{m+1}|\xi|^{-(m+1) / 2} t^{(m+1) / 4}$ et cette majoration est en accord avec (1.7). Il reste à montrer que l'on peut majorer la somme des deux premiers termes par $C_{m+1} \Phi_{m+1}(|\xi|, t)$. Or, d'après (1.8),

$$
\begin{aligned}
& \lambda_{\infty} e^{-t|\xi|^{2}}|\widehat{\mu}(\xi)|+C_{m}^{\prime} \lambda_{1} \int_{0}^{t}|\xi| e^{-(t-s)|\xi|^{2}} \Phi_{m}(|\xi| / 2, s) d s \\
& \quad \leq e^{-t|\xi|^{2}}|\widehat{\mu}(\xi)|+C_{m}^{\prime} \sum_{k=0}^{m-1} \mathcal{M}\left(\xi / 2^{k+1}\right) \int_{0}^{t} \lambda_{1}^{k+1} e^{\left(-t+s-s / 4^{k+1}\right)|\xi|^{2}}|\xi| s^{k / 2} d s \\
& \leq e^{-t|\xi|^{2}}|\widehat{\mu}(\xi)|+C_{m}^{\prime \prime} \sum_{k=0}^{m-1} \lambda_{1}^{k+1} \mathcal{M}\left(\xi / 2^{k+1}\right) e^{-t|\xi|^{2} / 4^{k+1}} t^{(k+1) / 2} \leq C_{m+1} \Phi_{m+1}(\xi, t) .
\end{aligned}
$$

La démonstration de la proposition 1 est ainsi achevée. Comme nous l'avons déjà observé, ceci et les lemmes 2 et 3 impliquent immédiatement la conclusion iii) du théorème 1.

\section{Profiles de la vorticité pour $|x|+t \rightarrow \infty$}

La solution, locale en temps, du théorème 1 est en fait globale, si la vorticité initiale vérifie une condition de petitesse convenable. Ici nous allons étudier le comportement asymptotique de $\omega$ pour $x$ et/ou $t \rightarrow \infty$.

Nous commençons par un résultat très simple, dans lequel on exige peu de localisation spatiale pour la vorticité initiale. La proposition suivante s'inspire d'un résultat de [Mi] relatif au champ de vitesse, où on obtient la conclusion (2.2) ci dessous, sans faire appel à la vorticité.

Proposition 2 Soit $\mu$ un champ de vecteurs défini sur $\mathbb{R}^{3}$, tel que $\operatorname{div}(\mu)=0$ et $|\mu(x)| \leq c(1+|x|)^{-2}$. Il existe une constante absolue $\epsilon>0$ telle que si $\sup _{\mathbb{R}^{3}}|x|^{2}|\mu(x)|<$ $\epsilon$, alors il existe une solution globale $(u, \omega)$ de (EI), vérifiant

$$
\begin{array}{rlrl}
|\omega(x, t)| & \leq C(1+|x|)^{-2}, & & |\omega(x, t)| \leq C(1+t)^{-1}, \\
|u(x, t)| \leq C(1+|x|)^{-1}, & & |u(x, t)| \leq C(1+t)^{-1 / 2}
\end{array}
$$

et $\omega(0)=\mu$ au sens faible.

Dém. Il suffit de montrer (2.1), car (2.2) en est une conséquence immédiate. En effet, de $\omega(t) \in L^{3,1}\left(\mathbb{R}^{3}\right)$, on déduit

$$
\|u(t)\|_{\infty} \leq\|K\|_{L^{3 / 2, \infty}}\|\omega(t)\|_{L^{3,1}} \leq C\|\omega(t)\|_{L^{3 / 2, \infty}}^{1 / 2}\|\omega(t)\|_{\infty}^{1 / 2} \leq C t^{-1 / 2} .
$$


D'autre part, on a déjà observé que $\omega \in Y_{2}$ (uniformément en $t$ ) implique $|u(x, t)| \leq$ $C(1+|x|)^{-1}$.

À l'aide d'un simple changement d'échelle (rappelons que si $\omega(x, t)$ est une solution de (EI), alors $\lambda^{2} \omega\left(\lambda x, \lambda^{2} t\right)$ résout encore (EI)), nous pouvons supposer que $\sup _{x}\left(1+|x|^{2}\right)|\mu(x)|$ soit petit. Par conséquent, on peut trouver une constante $C>0$ telle que l'évolution linéaire vérifie $\left|e^{t \Delta} \mu(x)\right| \leq C \epsilon(1+|x|)^{-2}$ et $\left|e^{t \Delta} \mu(x)\right| \leq C \epsilon t^{-1}$. Alors, la conclusion sera une conséquence immédiate de l'algorithme (1.2), si nous montrons que $A(u, \omega)$ est borné dans l'espace induit par (2.1) et (2.2). Ceci est facile : commençons par remplacer $A$ par l'opérateur scalaire

$$
\tilde{A}(u, \omega)=\int_{0}^{t} \int f(x-y, t-s)(u \omega)(y, s) d y d s
$$

où $u$ et $\omega$ sont traitées ici comme deux fonctions scalaires et $f(x, t)$ se comporte comme les dérivées d'ordre 1 de la gaussienne $g_{t}(x)=(4 \pi t)^{-3 / 2} e^{-|x|^{2} / 4 t}$. On a, en particulier,

$$
\|f(\cdot, t)\|_{1}=c t^{-1 / 2} \quad \text { et } \quad|f(x, t)| \leq C_{\gamma} t^{(\gamma-4) / 2}|x|^{-\gamma} \quad \text { pour tout } \gamma \geq 0 .
$$

La première partie dans (2.4) implique $\|\tilde{A}(u, \omega)(t)\|_{\infty} \leq C t^{1 / 2}$. On déduit l'estimation $\sup _{x, t}|x|^{2}|\tilde{A}(u \omega)(x, t)|<\infty$ de (2.1), (2.2) et des propriétés de $f$, à l'aide du découpage $\tilde{A}(u, \omega)=I_{1}+I_{2}$,

$$
\begin{array}{ll}
\text { où } & I_{1} \equiv \int_{0}^{t} \int_{|y| \leq|x| / 2} f(x-y, t-s)(u \omega)(y, s) d y d s, \\
\text { et } & I_{2} \equiv \int_{0}^{t} \int_{|y| \geq|x| / 2} f(x-y, t-s)(u \omega)(y, s) d y d s .
\end{array}
$$

Ensuite, $\tilde{A}(u, \omega)(t)=J_{1}+J_{2}$, où

$$
J_{1} \equiv e^{t \Delta / 2} \tilde{A}(u, \omega)(t / 2) \quad \text { et } \quad J_{2} \equiv \int_{t / 2}^{t} f(t-s) *(u \omega)(s) d s .
$$

De $\sup _{t}\|\tilde{A}(u, \omega)(t)\|_{L^{3 / 2, \infty}}<\infty$, on déduit par dualité

$$
\left\|J_{1}(\cdot, t)\right\|_{\infty} \leq C\left\|g_{t}\right\|_{L^{3,1}} \leq C t^{-1}
$$

De (2.1), (2.2) et la première de (2.4), on a

$$
\left\|J_{2}(\cdot, t)\right\|_{\infty} \leq \int_{t / 2}^{t}(t-s)^{-1 / 2} s^{-3 / 2} d s \leq C t^{-1} .
$$

Cela termine la démostration de la proposition 2.

Lorsque la vorticité initiale est mieux localisée $\left(\mu \in Y_{\gamma}\right.$, avec $\left.\gamma>2\right)$ on obtiendra de profils de décroissance de la forme $\sup _{x, t}(1+|x|)^{\alpha}|\omega(x, t)| \leq C_{\alpha}(t)$, pour tout $0 \leq \alpha \leq \gamma$.

Avant de donner une formulation plus précise de ce résultat, observons qu'il n'est pas raisonnable de s'attendre à ce que $\omega(x, t)$ ait une décroissance rapide, à la fois 
en $x$ et $t$. En effet, même si l'on suppose $\mu \in \mathcal{S}\left(\mathbb{R}^{3}\right)$ et que tous les moments de $\mu$ soient nuls, une telle propriété serait fausse, en général, pour l'évolution linéaire $e^{t \Delta} \omega\left(x, t_{0}\right)$, pour tout $t_{0}>0$ fixé. Ceci est lié au phénomène de perte instantanée de cancellations de $\omega$, que l'on a déjà mentionné dans la section 1 .

Cependant, on peut contrôler l'explosion à l'infini des constantes $C_{\alpha}(t)$, comme le théorème suivant (qui contient, en particulier, le résultat de la proposition 2) le montre.

Théorème 2 Soient $\gamma \geq 2(\gamma \neq 3,4)$, $\mu$ un champ de vecteurs à divergence nulle, tel que $|\mu(x)| \leq C(1+|x|)^{-\gamma}$. On peut trouver une constante absolue $\epsilon>0$ telle que, si $\sup |x|^{2}|\mu(x)|<\epsilon$,

i) Il existe une solution $\omega(x, t)$ de (EI) vérifiant

$$
|\omega(x, t)| \leq C_{\alpha}(1+|x|)^{-\alpha}(1+t)^{-\beta / 2} \quad(0 \leq \alpha \leq \gamma, \alpha+\beta=\min \{\gamma, 4\}) .
$$

ii) Si $\gamma>4(\gamma \neq 5)$ et $\int x_{j} \mu(x) d x=0(j=1,2,3)$, alors (2.5) est améliorée par

$$
|\omega(x, t)| \leq C_{\alpha}(1+|x|)^{-\alpha}(1+t)^{-\beta / 2} \quad(0 \leq \alpha \leq \gamma, \alpha+\beta=\min \{\gamma, 5\}) .
$$

Ces conclusions restent valables pour $\gamma=3,4,5$, pourvu que la solution de l'équation de la chaleur avec donnée initiale $\mu$ vérifie $\sup _{x, t}(1+|x|)^{\gamma}\left|e^{t \Delta} \mu(x)\right|<\infty$ et $\sup _{x, t}(1+t)^{\gamma / 2}\left|e^{t \Delta} \mu(x)\right|<\infty$.

Dém. On peut se limiter à $|x| \geq 1$ et $t \geq 1$. Commençons par traiter le cas $2<$ $\gamma<3$. Nous allons montrer que les solutions approchées données par l'algorithme (1.2) sont localisées, lorsque la vorticité initiale est localisée. Plus précisément, nous allons prouver que $(1+|x|)^{\gamma}\left|\omega^{j}(x, t)\right| \leq M_{j, \gamma}(j=0,1, \ldots)$ et $\sup _{j} M_{j, \gamma} \equiv M_{\gamma}<\infty$. Cette démarche s'inspire de [Ka84], où l'on traite le cas du champ de vitesse et la localisation est mesurée par l'appartenance aux espaces $L^{p}(p>1)$.

En effet, on procède par récurrence, en utilisant la version scalaire (2.3) de l'opérateur bilinéaire $A(u, \omega)$ : évidemment $\left|\omega^{0}(x, t)\right|=\left|e^{t \Delta} \mu(x)\right| \leq M_{0, \gamma}(1+|x|)^{-\gamma}$. D'après la démonstration de la proposition 2, on peut trouver une constante $c>0$ telle que $\left|u^{j}(x, t)\right| \leq c \epsilon t^{-1 / 2}$ pour tout $j=0,1, \ldots$. Alors, grâce à (2.4),

$$
\begin{array}{r}
\left|\tilde{A}\left(u^{j}, \omega^{j}\right)(x, t)\right| \leq C \epsilon M_{j, \gamma} \int_{0}^{t} \int_{|y| \leq|x| / 2}|x-y|^{-3}(t-s)^{-1 / 2} s^{-1 / 2}(1+|y|)^{-\gamma} d s d y \\
+C \epsilon M_{j, \gamma}\left(\int_{0}^{t}\|f(t-s)\|_{1} s^{-1 / 2} d s\right)(1+|x|)^{-\gamma} .
\end{array}
$$

Cela implique $M_{j+1, \gamma} \leq M_{0, \gamma}+C_{\gamma} \in M_{j, \gamma}$. La loi de Biot-Savart implique alors $|u(x, t)| \leq C_{\gamma}^{\prime}(1+|x|)^{-\gamma+1}$.

Comme nous pouvons supposer $C_{\gamma} \epsilon<1$, on en déduit $M_{\gamma}<\infty$ et $\sup _{x, t}|\omega(x, t)| \leq$ $M_{\gamma}(1+|x|)^{-\gamma}$. Ici $C_{\gamma} \rightarrow \infty$ pour $\gamma \rightarrow 3$ mais, comme nous allons le voir dans un instant, il suffit d'appliquer cet argument pour un $\gamma_{0}$ fixé, $\left(2<\gamma_{0}<3\right)$. On peut alors imposer une condition de petitesse indépendante de de $\gamma$. 
D'autre part, nous savons que $\|\omega(t)\|_{\infty} \leq C(1+t)^{-1}$ et $\|u(t)\|_{\infty} \leq C(1+t)^{-1 / 2}$. Afin d'améliorer ces estimations, on observe que $\|u \omega(t)\|_{1} \leq C_{\delta}(1+t)^{-\delta}$, pour tout $0 \leq \delta<(3 \gamma-6) /(2 \gamma-1)$. On fixe maintenant un réel $a(0<a<1)$, que l'on choisira dans un instant. Ensuite,

$$
\|\tilde{A}(u, \omega)(t)\|_{\infty} \leq \int_{0}^{t-t^{a}}(t-s)^{-2}\|u \omega(s)\|_{1} d s+\int_{t-t^{a}}^{t}(t-s)^{-1 / 2}\|u \omega(s)\|_{\infty} d s .
$$

La première intégrale est majorée par $C_{\delta} t^{-2 a+1-\delta}$ et la deuxième par $C t^{-3 / 2+a / 2}$. On choisit $a=1-2 \delta / 5$, avec $0 \leq \delta<(3 \gamma-6) /(2 \gamma-1)$. Dans ce cas, $\|\tilde{A}(u, \omega)(t)\|_{\infty} \leq$ $C_{\beta} t^{-\beta / 2}$, pour tout $2 \leq \beta<(26 \gamma-22) /(10 \gamma-5)$.

D'autre part, $\left\|e^{t \Delta} \mu\right\|_{\infty} \leq C_{\gamma} t^{-\gamma / 2}$ et cette estimation est meilleure que celle que nous avons obtenue jusqu'ici pour $\|\tilde{A}(u, \omega)\|_{\infty}$. D'où, $\|\omega(t)\|_{\infty} \leq C_{\beta}(1+t)^{-\beta}$ $(2 \leq \beta<(26 \gamma-22) /(10 \gamma-5))$ et, par conséquent, $\|u(t)\|_{\infty} \leq C_{\beta}(1+t)^{-(\beta-1) / 2}$.

On pourra retenir la conclusion suivante : sous les hypothèses faites $(2<\gamma<3)$, il existe $\gamma_{0}\left(2<\gamma_{0} \leq \gamma\right)$ tel que $|\omega(x, t)| \leq C(1+|x|)^{-\gamma_{0}}$ et $\|\omega(t)\|_{\infty} \leq C(1+t)^{-\gamma_{0} / 2}$. En outre, on a les mêmes estimations pour $u(x, t)$, quitte à remplacer $\gamma_{0}$ par $\gamma_{0}-1$. Il est maintenant facile (en revenant aux termes $I_{1}, I_{2}, J_{1}$ et $J_{2}$ introduits dans la démonstration de la proposition 2) de voir que ces estimations restent valables pour $\tilde{A}(u, \omega)$, avec un exposant $\gamma_{1}>\gamma_{0}$. À l'aide d'un argument de boot-strap, on voit donc que $\|\omega(t)\|_{\infty} \leq C_{\gamma} t^{-\gamma / 2}$.

On traite maintenant le cas $3 \leq \gamma<4$. Pour ce faire, on revient encore une fois à $I_{1}$ et $I_{2}$. De (2.4) et du résultat montré à l'étape précédente, on déduit immédiatement $I_{2} \leq C_{\delta}(1+|x|)^{-\delta}$ pour tout $0 \leq \delta<4$. Donc $I_{2} \leq C_{\gamma}(1+|x|)^{-\gamma}$. De même, $I_{1} \leq C_{\gamma}|x|^{-\gamma}$.

À l'aide d'un argument de dualité, on voit alors que $\left\|J_{1}(t)\right\|_{\infty}$ est majoré par $C_{\gamma} t^{-\gamma}$. En outre, $\left\|J_{2}(t)\right\|_{\infty} \leq C_{\delta}(1+t)^{-\delta / 2}(0<\delta<4)$. Ces estimations impliquent immédiatement (2.5).

La démonstration de i), dans le cas $\gamma \geq 4$, utilise un argument de boot-strap: grâce aux mêmes techniques utilisées ci-dessus, on montre sans peine que si l'on dispose d'une majoration de la forme $|\omega(x, t)| \leq C_{\alpha}(1+|x|)^{-\alpha}(1+t)^{-(4-\alpha) / 2}$, pour un $\delta \geq 3$ et tout $\alpha$ tel que $0 \leq \alpha \leq \delta$, alors on a, en fait, $|\tilde{A}(u, \omega)(x, t)| \leq$ $C_{\alpha}(1+|x|)^{-\alpha}(1+t)^{-(4-\alpha) / 2}$, pour tout $0 \leq \alpha \leq \delta+1$. Après un nombre fini d'iterations on trouve bien (2.5).

La démonstration de la deuxième partie du théorème est facile : sous les hypothèses faites on voit immédiatement que $\left|e^{t \Delta} \mu(x)\right|$ est majoré par le terme de droite dans (2.6). En outre, de $\omega=\nabla \wedge u$, $\operatorname{div}(u)=0$ et des résultats de décroissance pour $|x| \rightarrow \infty$ obtenues dans la conclusion i) du théorème, on voit que $\int x_{j} \partial_{h}\left(u_{h} \omega-\omega_{h} u\right) d x=0(j=1,2,3)$. Grâce à cette cancellation supplémentaire, une intégration par parties dans l'intégrale de convolution définissant $A(u, \omega)$ nous conduit à considérer une variante de l'opérateur $\tilde{A}$. Ce nouveau opérateur est défini comme $\tilde{A}$, à ceci près que le noyau $f$, cette fois-ci, se comporte comme les dérivées d'ordre 2 de la gaussienne. Les modifications à apporter à la démonstration sont triviales. 


\section{Une classe de tourbillons plus oscillants}

L'estimation (2.6) est optimale, au moins dans le cas générique. Cependant, la démonstration du théorème 2 montre que l'on pourrait faire mieux, si l'on disposait des cancellations supplémentaires $\int|x|^{\alpha} \mu(x) d x=0$ et $\int x^{\alpha} \partial_{h}\left(u_{j} \omega_{h}-\omega_{j} u_{h}\right)(x, t) d x \equiv 0$, pour tout $t \geq 0,|\alpha|=2$, et $j=1,2,3$ (dans ce cas, alors, les moments d'ordre 2 de $\mu$ seraient conservées).

En fait, il est alors facile de construire une classe exceptionnelle de solutions avec ces propriétés. Pour ce faire, il suffit de considérer les tourbillons associés aux champs de vitesse symétriques introduits dans [Bra01].

Un écoulement symétrique est caractérisé par les propriétés suivantes :

i) $\omega_{1}\left(x_{1}, x_{2}, x_{3}, t\right)=\omega_{2}\left(x_{3}, x_{1}, x_{2}, t\right)=\omega_{3}\left(x_{2}, x_{3}, x_{1}, t\right)$

ii) $\omega_{j}(x, t)$ est paire en $x_{j}$ et impaire en $x_{k}(j, k=1,2,3$ et $j \neq k)$.

Alors, $u=K * \omega$ vérifie également la conditions i), et la condition ii) avec la parité/imparité invertie).

Si on revient à la construction de la solution $(\omega, u)$ obtenue dans la proposition 2 , on montre sans difficultés que, si $\mu$ vérifie i) et ii) pour $t=0$, alors il en est de même pour $\omega(t)$, pour tout $t>0$.

Supposons maintenant que $\mu$ soit suffisament localisée : $|\mu(x)| \leq C(1+|x|)^{-\gamma}$, avec $\gamma>5$. Si $\mu$ vérifie ii) pour $t=0$, alors, évidemment, $\int x^{\alpha} \mu(x) d x=0(0 \leq|\alpha| \leq$ $1)$. D'autre part, comme div $\mu=0$, on a $\int\left(x_{2} x_{3} \mu_{1}(x)+x_{3} x_{1} \mu_{2}(x)+x_{1} x_{2} \mu_{3}(x)\right) d x=$ 0 (voir, par exemple [GW]). Si $\mu$ vérifie aussi $i)$, on voit alors les moments d'ordre 2 de $\mu$ sont nuls également. Si, de plus, $\gamma>6$ les moments de $\mu$ sont nuls jusqu'à l'ordre 3.

Par conséquent, sous les hypothèses faites, $\left|e^{t \Delta} \mu(x)\right|$ est majoré par

$$
C_{\alpha}(1+|x|)^{-\alpha}(1+t)^{-\beta / 2} \quad(0 \leq \alpha \leq \gamma, \quad \alpha+\beta=\min \{\gamma, 6\}),
$$

voire même par

$$
C_{\alpha}(1+|x|)^{-\alpha}(1+t)^{-\beta / 2} \quad(0 \leq \alpha \leq \gamma, \quad \alpha+\beta=\min \{\gamma, 7\}),
$$

si $\gamma>6$.

Pour démontrer que la solution $\omega(x, t)$ elle même est majorée par (3.1) ou (3.2), il suffit alors de montrer que

$$
\int x^{\alpha} \partial_{h}\left(u_{h} \omega-\omega_{h} u\right)(x, t) d x \equiv 0,
$$

respectivement, pour $|\alpha|=2$, ou $|\alpha| \leq 3$, et de proceder ensuite comme dans la démonstration du théorème 2 .

Les cancellations (3.3) sont triviales pour $|\alpha|=3$ ou bien $|\alpha|=2$ et $x^{\alpha}$ de la forme $x_{j}^{2}(j=1,2,3)$. Les autres cas nécessitent d'un petit calcul : nous pouvons choisir, par exemple, $j=1$ et $\alpha=(0,1,1)$. Il s'agit de montrer que $\int x_{2} x_{3} \partial_{2}\left(u_{1} \omega_{2}-u_{2} \omega_{1}\right) d x+\int x_{2} x_{3} \partial_{3}\left(u_{1} \omega_{3}-u_{3} \omega_{1}\right) d x=0$. En effet, d'après la définition de $\omega$ et $\operatorname{div}(u)=0$, cette expression vaut $\int \frac{1}{2}\left(u_{2}^{2}-u_{3}^{2}\right) d x+\int\left(x_{3} u_{2} \partial_{3} u_{2}+\right.$ 
$\left.x_{3} u_{1} \partial_{3} u_{1}-x_{2} u_{3} \partial_{2} u_{3}-x_{2} u_{1} \partial_{2} u_{1}\right) d x$. D'après la condition i), ces deux intégrales sont bien nulles.

Cette discussion nous amène au résultat suivant.

\section{Théorème 3}

i) Si $\mu$ est symétrique et vérifie les conditions du théorème 1 avec $\gamma>5(\gamma \neq 6)$, alors l'estimation (2.5) est valable pour tout $\alpha$ et $\beta$ tels que $0 \leq \alpha \leq \gamma$ et $\alpha+\beta=$ $\min \{\gamma, 6\}$.

ii) Si de plus $\gamma>6$ et $(\gamma \neq 7)$, alors on peut prendre $\alpha+\beta=\min \{\gamma, 7\}$. En outre, ces conclusions restent valables pour $\gamma=6,7$, pourvu que $\sup _{x, t}(1+|x|)^{\gamma}\left|e^{t \Delta} \mu(x)\right|<\infty$.

Il est à noter que, lorsque les cancellations (3.3) sont satisfaites pour tout $t \geq 0$, et pour tout multi-indice $\alpha$, avec $|\alpha| \leq m(m \in \mathbb{N})$, les moments de $\mu$ sont conservés au cours du temps, jusqu'à l'ordre $m$. À l'heure actuelle, nous ne connaissons aucun exemple de telle situation, en dehors de celle correspondente aux tourbillons symétriques (qui ne s'applique, en général, que pour $m=0,1,2,3$ ).

On peut construire, par ailleurs, un exemple d'écoulement symétrique tel que $\omega(t)$ appartient à la classe de Schwartz pour tout $t \geq 0$ et tel que

$$
\forall \eta>0 \quad \sup _{x, t}(1+|x|)^{7+\eta}|\omega(x, t)|=+\infty .
$$

Pour cet écoulement, les moments d'ordre 4 de $\mu$ sont nuls, mais cette propriété est instantanément perdue au cours de l'évolution. Par conséquent, l'estimation du théorème 3 est aussi optimale.

Nous faisons remarquer que l'application récente la théorie des variétés invariantes aux équations de Navier-Stokes a permis de démontrer l'existence de solutions telles que $\|\omega(t)\|_{\infty}$ décroit vers 0 avec un taux algébrique fixé, mais arbitrairement élevé (voir [GW]). Cependant, ces méthodes ne produisent aucun exemple explicite de données initiales conduisant à de telles solutions.

Une quantité considérable d'articles a été consacrée à l'étude de la décroissance à l'infini de l'énergie $\|u(t)\|_{2}^{2}$ du fluide (voir, par exemple, [Sch], [Wie] et la bibliograhie de $[\mathrm{GW}])$. Les écoulements symétriques sont les premiers exemples connus de solutions dans $\mathbb{R}^{3}$ telles que $\|u(t)\|_{2}^{2}=o\left(t^{-5 / 2}\right.$ ), pour $t \rightarrow \infty$ (de tels exemple sont connus depuis longtemps dans $\mathbb{R}^{2}$, cf. [Sch]). Le théorème 3 implique immédiatement que, pour ces écoulements symétriques, on peut avoir $\|u(t)\|_{2}^{2}=O\left(t^{-\beta}\right)$, pour tout $\beta<9 / 2$. En fait, on peut atteindre la valeur limite $9 / 2$ (et sans restrictions sur la vorticité), en utilisant les techniques développées par M. Schonbek et M. Wiegner dans le cadre des solutions faibles. Nous renvoyons à [Bra02] pour plus de détails.

\section{References}

[Bra01] L. Brandolese, On the Localization of Symmetric and Asymmetric Solutions of the Navier-Stokes Equations dans $\mathbb{R}^{n}$, C. R. Acad. Sci. Paris, t. 332, Série I, 125-130 (2001). 
[Bra02] L. Brandolese Asymptotic behavior of the energy and pointwise estimates for solutions to the Navier-Stokes equations, prépublication.

[Da] R. Danchin, Analyse numérique et harmonique d'un problème de mécanique des fluides, Thèse de doctorat, École Polytechnique, Palaiseau, France, Décembre (1996).

[DS] Dobrokhotov S. Y., Shafarevich, A. I., Some Integral Identities and Remarks on the Decay at Infinity of the Solutions to the Navier-Stokes Equations in the Entire Space, Russ. J. Math. Phys. Vol. 2, N. 1 (1994) 133-135

[GM] Y. Giga, T. Miyakawa, Navier-Stokes Flow in $\mathbb{R}^{3}$ with Measures as Initial Vorticity and Morrey Spaces, Commun. in Partial Diff. Equations, 14, 5, 577618, (1989).

[GW] T. Gallay, C. E. Wayne, Long-time asymptotics of the Navier-Stokes and vorticity equations on $\mathbb{R}^{3}$, prépublication, Univ. Orsay (2001).

[Ka84] T. Kato, Strong $L^{p}$-solutions of the Navier-Stokes equations in $\mathbb{R}^{m}$, with applications to weak solutions, Math. Zeit. 187, 471-480 (1984).

[LT] Lu Ting, On the application of the integral invariants and decay laws of vorticity distributions, J. Fluid Mech. 127, 497-506 (1983).

[Mi] T. Miyakawa, On space time decay properties of nonstationary incompressible Navier-Stokes flows in $\mathbb{R}^{n}$, Funkcial. Ekvac., 32, N. 2, 541-557 (2000).

[Sch] M. E. Schonbek, Lower Bounds of Rates of Decay for Solutions to the NavierStokes Equations, J. of the Amer. Math. Soc., 4, N. 3, 423-449 (1991).

[Wie] M. Wiegner, Decay Results for Weak Solutions of the Navier-Stokes Equations on $\mathbb{R}^{n}$, J. London Math. Soc., 2, N. 35, 303-313 (1987).

ae

Centre de Mathématiques et de Leurs Applications,

ENS DE CACHAN, 61, AV. DU PRÉSIDENT Wilson

94235 Cachan Cedex.

brandole@cmla.ens-cachan.fr 